\title{
A WEB-BASED WIRELESS ACCESS TO ONLINE INFORMATION SYSTEM FOR NAFDAC
}

\author{
Calister N. $\mathrm{Mba}^{1}$ and Prof. Hyacinth C. Inyama ${ }^{2}$ \\ ${ }^{1}$ Department of Computer Engineering, Caritas University, Amorji-Nike, Enugu. \\ Enugu State, Nigeria. \\ mbacally@gmail.com \\ ${ }^{2}$ Department of Electronic and Computer Engineering, Nnamdi-Azikiwe University, \\ Awka, Anambra State, Nigeria. \\ hcinyiama2002@yahoo.com
}

\begin{abstract}
Wireless access technology has made much impact in the growth and development of corporate information systems. This paper presents how a web-to-database production tool was used to achieve a real time wireless access to NAFDAC corporate database. An online version of the database was created using MySQL along with both client and server side software that capture queries from and generate reports to remote mobile monitoring operator devices in real time. The client sends a request using a web browser in the WAP (Wireless Application Protocol) enabled mobile device and receives a response from the database. This is done by a web server, which supplies the response in a HTML format to the user's mobile device. The system architecture and the developed software are presented. The system was implemented as an experimental set up in a laboratory.
\end{abstract}

\section{KEYWORDS}

NAFDAC, MySQL, Web server, WAP, HTML, Wireless access, Web-to-middleware.

\section{INTRODUCTION}

Secure and efficient communication between field monitoring operators, the general public and the NAFDAC Database server is critical for smooth and efficient control of their activities in real time, through mobile phones. "Tongtong et al (2012) reported that along with the rapid development in wireless technologies today, people can receive high speed multimedia information service at any place covered by a communication network." "Haydyn (2004) reported that long distance information exchange through Wide Area Network (WAN) has become well developed for information transmission or acquisition." This advance in WAN and LAN technologies, are now able to support scalable multimedia services. "Hela Limam et al (2011) reported that with the advance of Web Services technologies and the emergence of Web Services into the information space, tremendous opportunities for empowering users and organizations appear in various application domains including electronic commerce, travel, intelligence information gathering and analysis, health care, digital government, etc." 
International Journal of Database Management Systems ( IJDMS ) Vol.4, No.6, December 2012

"Hela also reported that the emergence of Web Services Communities as a model for integrating heterogeneous web information has opened up new possibilities of interaction and offered more potential for interoperability"

"Zhang et al (2007), Lu et al (2005) and Ramamurthy et al (2007) reported that the advantages of wireless technology which include reliability, simplicity and low cost in both installation and maintenance have been extensively exploited in real time monitoring and control of various areas of agriculture, engineering and industrial processes respectively." Inspired by the observation above, this work seeks to implement a web enabled smart system that can achieve online, real time authentication of drugs and off the shelf food items under NAFDAC administration, through a secure two way communication between a smart phone and the NAFDAC online database computer. Our major challenge in this work has been to determine the appropriate technique suitable for NAFDAC and how to use such technique securely, with good performance. We opted to connect directly to the database server through a Virtual Private Network (VPN) connection on a public Internet. "Mark (2008) reported that apart from administering logins to the database, data retrieval from the SQL propriety format can be very fast especially, as a remote field operator in NAFDAC or a client does not need to share the data with any other applications." The conflict with other users is avoided. Mohamed et al (2011) studied how to simplify querying and manipulating temporal facts in SQL3, using a model that integrates time in a native manner. They proposed temporal extension to the standard SQL3 by new keywords, which are used to define several versions of temporal operators to SQL, and then to perform many "forms" of querying and updating temporal databases. Radha et al (2011) reported that indexing technique greatly reduces the overall storage size required and also provides reduced execution time, which proves the efficiency of the indexing technique for efficient database querying. Ines et al (2012) observed that in traditional Database Management System, the user's query is treated at only syntactical level which implies that the answer to the query is highly dependent on his level of knowledge about the content. He proposed two approaches based on domain ontology constructed from the database schema to overcome this limitation and improve the answers of the database queries by detecting and resolving naming and schema conflicts. The use of SQL client classes can be implemented in both the full .NET framework and the .NET compact framework, thus expanding their use to mobile devices. This exactly serves our major purpose.

\section{SYSTEM ARCHITECTURE}

Figure 1 depicts an integration of two different options available to the users for accessing online database information. The two options include using either a WAP or non-WAP enabled GSM handset to access information from NAFDAC online information system. With the first option, when a request is made from any of the WAP enabled handsets, the Internet captures the request and forwards it to the server side software, which accesses the requested information via MySQL database server. When the database server receives the request, it supplies the appropriate response which now goes back to the user that made the request through the same wireless Internet connectivity. Here, the client side software is not involved, since the handsets are WAP enabled. 


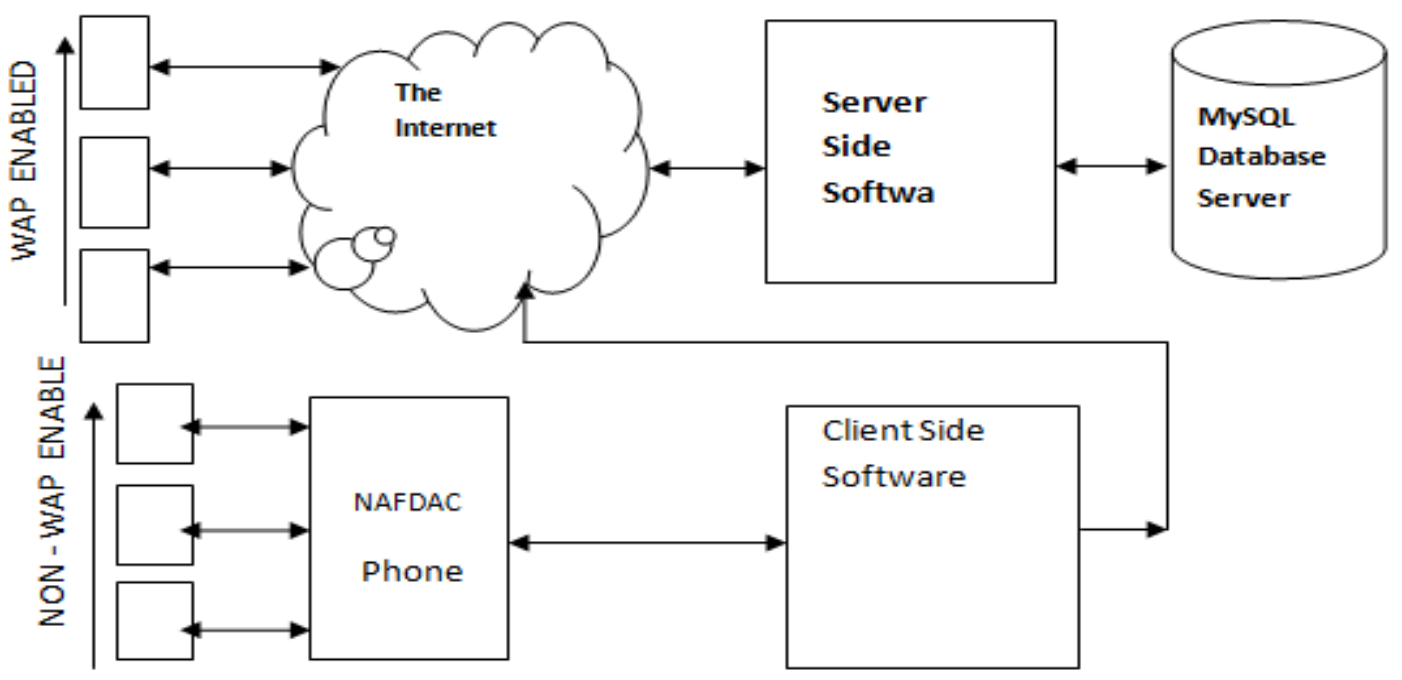

Figure 1: Online wireless access with both WAP and non -WAP enabled mobile phones.

With the second option, the handsets involved are not WAP enabled; therefore users can only access the software through the client side software resident at NAFDAC. The GSM (Global System for mobile Communication) handset at NAFDAC is connected to the computer system such that it can receive requests in form of text messages from other handsets and pass the received data to the client side software located at NAFDAC. The connection between the GSM handset and the computer system could be by a means of Bluetooth technology or by using a data cable with a software driver like the Nokia PC suite. The client side software then decodes the request from the customer or user and forwards an appropriate request for information storage or retrieval (as appropriate) to the server side software via the Internet. This software interacts with MySQL server to conclude the operation desired and communicates an appropriate response to the customer or user via the same route.

\subsection{Accessing Online Database Application Using MySQL}

\subsubsection{Internet Databases}

The generation, accessing and circulation of information have been greatly improved by the TCP/IP network popularly called the Internet. The following improvements have been made possible; the web's ability to access local and remote data in a database system, the simplicity of the interface, the cost effectiveness and of course, the compatibility and inter-operability of such data and the devices involved in the exchange. Generally the web has established a new high standard of information distribution.

\subsubsection{Server Side Extensions}

All Internet services, including access to online database are generally done through the web server. When an end user requests a web page using a web browser, the web server receives the page request, locates it from the cache, the RAM or the hard disk as the case may be. If the request page is found, it is sent back to the client (user) that made such request. Unfortunately, connecting to and reading from an online database are still impossible tasks for web browsers and web servers. There is therefore need to extend the web server's capability to enable it understand 
International Journal of Database Management Systems ( IJDMS ) Vol.4, No.6, December 2012

and process database queries and other requests. This will enable the web server support such services as database queries. Peter et al (2007) reported that this can be achieved through a server side extension. A server side extension is a program that interacts directly with the web server to handle specific types of requests. The server side extension program retrieves data from database and passes the retrieved data to the web server, which then forwards the data to the client's browser for display. A server side extension program is also known as web-to-database middleware.

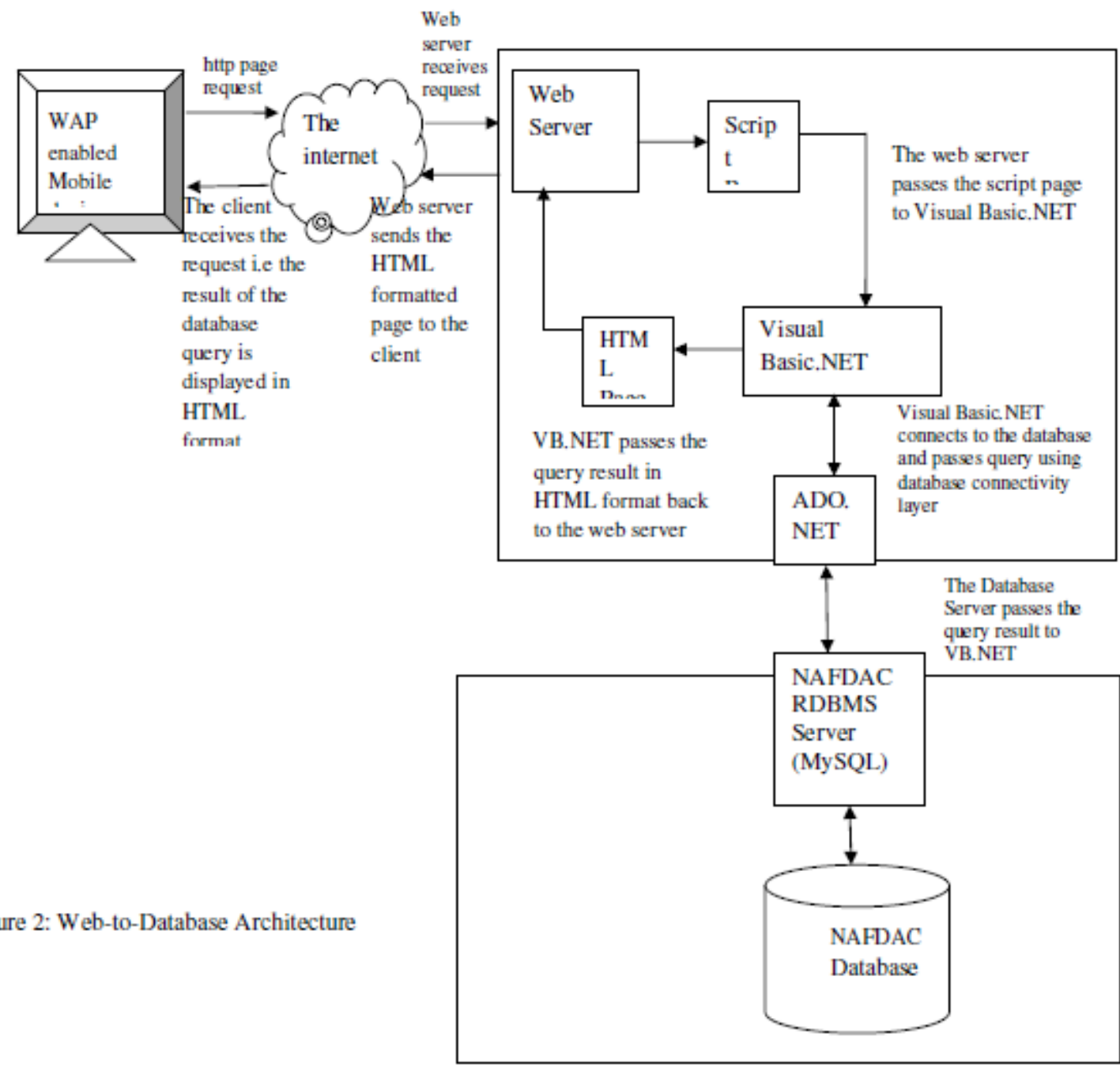

From figure 2, the following are observed:

(i) A NAFDAC client sends a request page (which usually has some scripting languages) to the web server using a client browser.

(ii) The web server receives and validates the request.

(iii) Visual Basic.Net reads, validates and executes the script. It connects to the database using the 
ADO.NET object model which provides an API (Application Programming Interface) for accessing the MySQL DBMS (Database Management System) programmatically, and passes the query using the database connectivity layer. ADO.NET (Active X Data Objects) was created for the .NET framework to replace Microsoft Active X Data Objects (ADO) technology.

(iv) The database server executes the query and passes the result back to Visual Basic.NET

(v)The Visual Basic.NET compiles the result set, dynamically generates the HTML formatted page that includes the query result from the database, and sends it to the web server.

(vi) The web server returns the HTML formatted page to the client browser.

(vii) The client browser displays the page on the client's WAP enabled mobile device.

\section{SOFTWARE ARCHITECTURE}

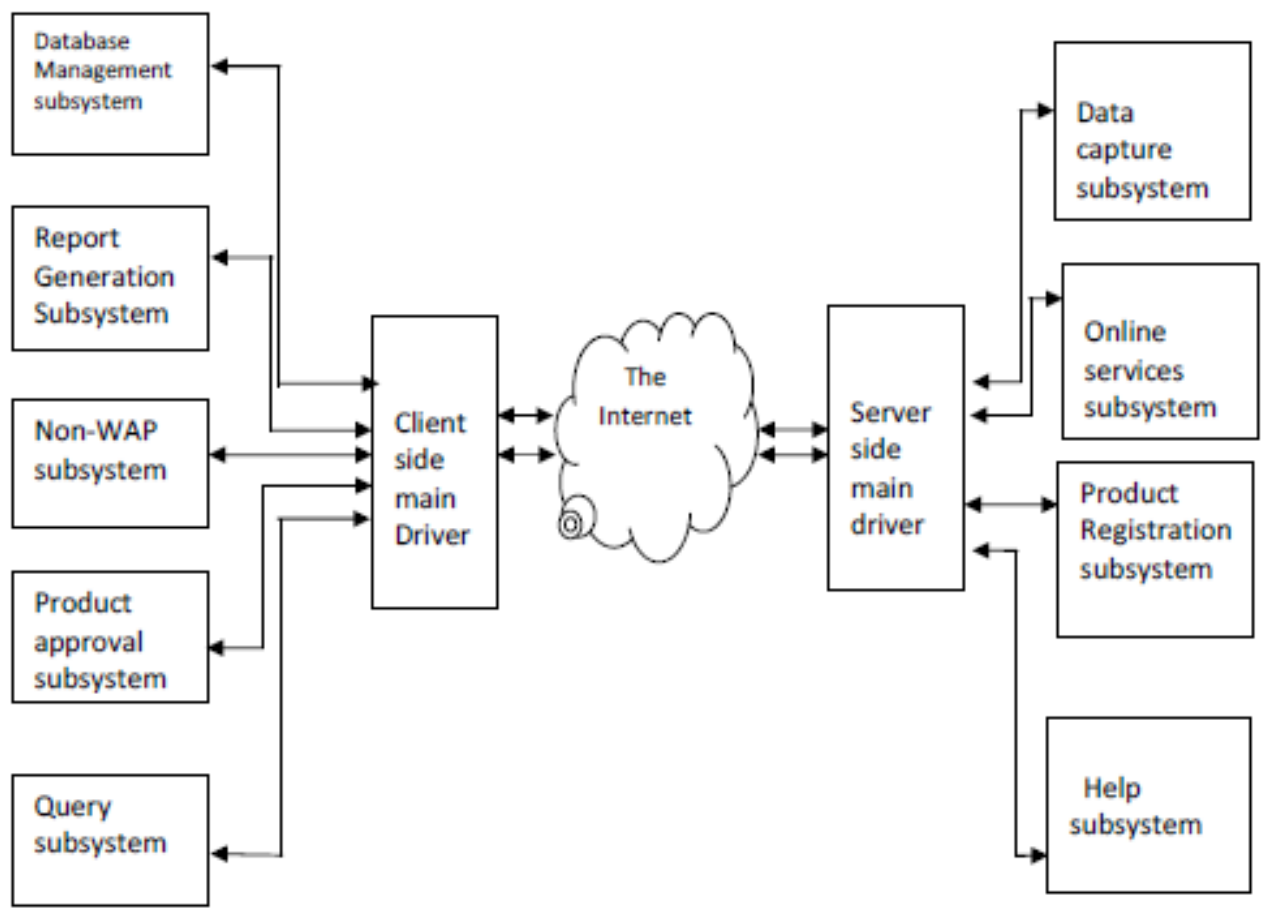

Figure 3: The software Architecture

The software was designed into two major parts viz: the client side and the server side. Each of these parts was designed into subsystems which were also broken into modules using a top down design approach. The presence of the main driver on doth sides makes it possible to access the subsystems and the modules. 
International Journal of Database Management Systems ( IJDMS ) Vol.4, No.6, December 2012

\subsection{The Client Side Software:}

The client side software contains all the subsystems and modules of the NAFDAC software. It is resident at NAFDAC such that NAFDAC IT (Information Technology) staff can access the entire system. Once a login is successful, either as staff or member, access to the client main menu is given. The client side software is a mirror image of the server side in addition to some subsystems which can only be accessed by NAFDAC and some law enforcement agencies. The subsystems in the client side include the Product Registration, Database Management, Data Capture, Online Services, Report Generation, Query, Product Approval, Non-WAP Cell Phone and the Help Subsystems.

\subsection{The Server Side Software}

The server side software consists of the subsystems that can be accessed through the Internet. There is a mirror image of these subsystems at the Client Side. The sever side is resident on the Internet and therefore gives open access to all users. Users with WAP enabled mobile devices access the server side by simply sending and sms (simple message signal) directly to the Internet, which also responds directly to the user. But users with non-WAP enabled mobile device access the server side software by sending sms to the GSM handset attached to the computer system resident at NAFDAC (i.e the client side computer). This goes ahead to the server side software through the Internet to query the database and returns the reply to the corresponding user through the same route. The server side software consists of the Data Capture, Online Services, Product Registration and Help subsystems. These subsystems and their modules are driven by the server side main driver as shown in figure 3 and the various subsystem drivers respectively.

\subsection{Database Design}

A NAFDAC database design was also part of the system design. The customized database which has some tables and forms was designed to capture and keep track of all data and information that enter the system as inputs /requests or output/response from/to users. The database was designed using MySQL. MySQL is an open source database management system commonly used in web applications due to its speed, flexibility and reliability. It employs SQL (Structured Query Language) for accessing and processing data contained in databases.

\section{CONCLUSION}

Information retrieval is a very crucial aspect of any information system. Improving the efficiency of any device is also a means of ensuring that the device resources are not wasted. The research has revealed that NAFDAC operations can be greatly enhanced by using mobile devices such as GSM handsets to access their database. In this case, there was a demonstration of how information can be retrieved from an online NAFDAC database using server side extension software. The inadequacy of mobile facilities required by NAFDAC for their inspection, verification, registration services among others can be overcome since they can use their personal mobile devices to access the NAFDAC database for such services. The impact of this user centric approach to accessing NAFDAC online database has been shown to bring improvement in the entire system. This is so because, instead of having just a centralized point for information verification, say in a zone or state, as currently in practice, several verification points can be made available within the cities and the rural localities. 
International Journal of Database Management Systems ( IJDMS ) Vol.4, No.6, December 2012

\section{REFERENCES}

[1] Haydyn A. Thompson, (2004)"Wireless and Internet Communication Technologies for Monitoring and Control”, Control Engineering Practice. (ELservier) vol 12 (6) P781-791, June, 2004.

[2] Hela Limam and Jalel Akaichi, (2011) 'Managing and Querying Web Services Communities: A Survey', International Journal of Database Management System (IJDMS) Vol3,No. 1 Feb 2011.

[3] Ines Fayech and Habib Ounalli, (2012) " Towards A Flexible Database Interrogation ', International Journal of Database Management System (IJDMS) Vol4, No. 3 June 2012.

[4] LU K.C, wang Y, Lyuch J.P, Lin P.Y, Loh C.H and Law K.H, (2005) "Application of Wireless Sensor for Structural Health Monitoring And Control," Eighteenth KKCNN Symposium on Civil Engineering. Dec 19-21 2005, Taiwan.

[5] Mark D. Sutton, (2008) "Direct Access to SQL Server Databases from Wireless Clients," Intel Software Networks. Sep 2008.

[6] Mohamed Mkaouar, Rafik Bouaziz, and Mohamed Moalla, (2011) " Querying and Manipulating Temporal databases', International Journal of Database Management System (IJDMS), Vol3,No. 1 Feb 2011.

[7] Peter Rob and Carlos Coronel, (2007) "Database System Design, Implementation and Management." Thomson Course Technology, 25 Thomson Place, Boston, Massachusetts. $7^{\text {th }}$ Edition.

[8] Radha Senthilkumar and Kannan Arputhara, (2011) 'Efficiently Querying the Indexed Compressed XML Data (IQX)', International Journal of Database Management System (IJDMS) Vol3, No 3 August 2011.

[9] Ramamurthy Harish, Prabu B.S, Gadh Rajit and Madni A.M, (2007) "Wireless Industrial Monitoring and Control Using Smart Sensor Platform," Accepted for publication in IEEE Sensors Journal.

[10] Tongtong Li, Jain Ren, and Xia ochen Tang, (2012) "Secure Monitoring and Control System for Smart Grid and Smart Home," IEEE Wireless Communication.

[11]ZHANG QIAN, Yang Ziang - long Zhou Yi-ming, Inang Li-ren and Guo xi-shan, (2007) “ A Wireless Solution For Green House Monitoring And Control System Based On ZIGBEE Technology,” Journal of Zhejiang University SCIENCE A 2007 8(10) PP 1584-1587.

\section{AUTHORS}

Engr Calister N.Mba is a Computer Scientist and Engineer, She is a Lecturer in the Department of Computer Engineering, Caritas University, Amorji-Nike, Enugu, Nigeria. She has a Barchellor of Engineering Degree (B.Eng.) in Computer Engineering and a Master of Science Degree (M.Sc.) in Computer Science. She has research interests in data and information Networks.

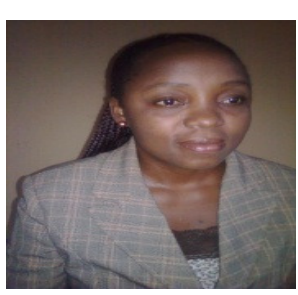

Prof. Inyiama Hyacinth Chibueze is a seasoned computer scientist and engineer, with a wealth of experience in both industry and academics. He is a professor in the Department of Electronic \& Computer Engineering, Nnamdi Azikiwe Federal University, Awka. Nigeria. His current research interest is using IT in solving human problems in industries with focus on Artificial Intelligence.

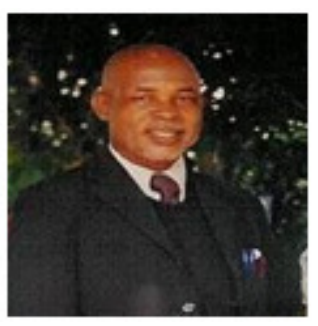

